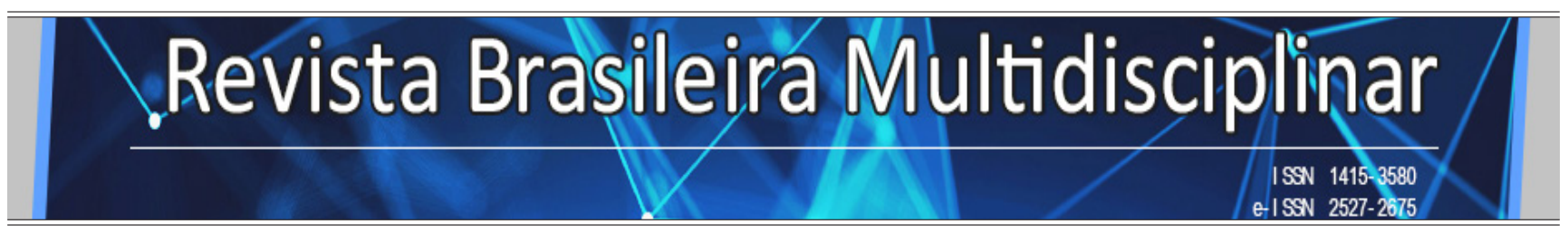

http://revistarebram.com/index.php/revistauniara

\title{
EMPREGO DA HOMEOPATIA PARA AFECÇÕES CUTÂNEAS DE RESULTADO INESTÉTICO: EVIDÊNCIAS DA LITERATURA
}

\author{
Bruna Galdorfini Chiari-Andréo*; Rualdo Valderrama Filho** \\ ${ }^{*}$ Docente da Universidade de Araraquara - UNIARA \\ ${ }^{*}$ Universidade de Araraquara - UNIARA \\ *Autor para correspondência e-mail:bgchiari-andreo@uniara.edu.br
}

\section{Palavras-chave}

\section{Homeopatia}

Cosméticos

Dermatologia

Pele

Efeitos inestéticos

\section{KEYWORDS}

Homeopathy

Cosmetics

Dermatology

Skin

Unaesthetic Effects

\section{RESUMO}

A homeopatia é uma terapia alternativa praticada há centenas de anos. É um tratamento baseado na lei dos semelhantes, pois utiliza substâncias que geram no corpo humano os mesmos efeitos causados pela patologia ou distúrbio que o acomete. Considerando o fato de que, a descrição dos sinais e sintomas produzidos pelos insumos ativos homeopáticos, muitas vezes, se assemelha às disfunções cutâneas, com resultados inestéticos, este trabalho propõe o uso da homeopatia, em formulações tópicas, associando o conhecimento tradicional da homeopatia com a tecnologia de cosméticos. Então, o objetivo foi realizar uma revisão da literatura buscando embasamento científico para o emprego da homeopatia para amenização de disfunções cutâneas que promovem impacto inestético. Foram realizadas buscas na literatura científica, utilizando-se documentos publicados entre 1993 e 2019. Foi possível listar insumos ativos homeopáticos que promovem efeitos semelhantes à acne, psoríase, caspa, alopecia, rosácea, dermatite atópica, sendo todas estas disfunções capazes de prejudicar a aparência do indivíduo, gerando inclusive impactos na qualidade de vida e na autoestima. A literatura relata diversos resultados positivos para controle destas alterações cutâneas com o uso da homeopatia.

\begin{abstract}
EMPLOYMENT OF HOMEOPATHY FOR CUTANEOUS AFFECTIONS OF ANESTHETIC RESULT: EVIDENCE OF LITERATURE

Homeopathy is an alternative therapy practiced for hundreds of years. It is a treatment based on the law of similars, since uses substances that generate in the human body the same effects caused by the pathology or disorder that affects it. Considering the fact that, the description of the signs and symptoms produced by the active homeopathic ingredients, often resembles cutaneous dysfunctions, with unsightly results, this study proposes the use of homeopathy, in topical formulations, associating the traditional knowledge of homeopathy with cosmetic technology. So, the aim was to carry out a literature review seeking a scientific basis for the use of homeopathy to alleviate skin dysfunctions that promote aesthetic impact. Searches were carried out in the scientific literature, using documents published between 1993 and 2019. It was possible to list active homeopathic ingredients that promote effects similar to acne, psoriasis, dandruff, alopecia, rosacea, atopic dermatitis, all of which are dysfunctions capable of impairing the appearance of the individual, even generating impacts on quality of life and self-esteem. The literature reports several positive results for controlling these skin changes with the use of homeopathy.
\end{abstract}




\section{INTRODUÇÃo}

A homeopatia é um tipo de terapia alternativa praticada há centenas de anos. Foi criada pelo médico alemão Cristiano Frederico Samuel Hahnemann e baseia-se na lei dos semelhantes, ou seja, uma determinada substância que pode provocar, em um indivíduo saudável, determinados sinais e sintomas de alguma disfunção ou patologia, pode ser capaz de promover a cura, se utilizada em um indivíduo doente em doses infinitesimais (MERRELL; SHALTS, 2002).

Apesar de a farmacopeia homeopática brasileira descrever a possibilidade de manipulação de formulações homeopáticas de uso tópico, como cremes e géis, estes tipos de formas farmacêuticas não são rotineiramente empregados sendo, muitas vezes, desconhecida a possibilidade de uso através destes excipientes pela população. Além disso, a homeopatia é compreendida, basicamente, como uma alternativa para o tratamento, alívio ou prevenção de patologias, sendo que neste sentido a utilização por via oral é a mais empregada e também parece ser a mais adequada. Mas, considerando a possibilidade de emprego de formas farmacêuticas de uso no setor cosmético, como cremes e géis, além de outras formas de uso tópico, para carrear insumos ativos homeopáticos, este trabalho visa avaliar se é possível o emprego da homeopatia para a amenização de disfunções de resultado inestético.

Adiciona-se ao exposto, o fato de que diversos tipos de disfunções cutâneas ainda são pouco compreendidas e com poucas opções de tratamentos. Dentre os tratamentos disponíveis, os retinoides e corticoides são extensivamente utilizados, estando associados a diversos riscos e efeitos adversos.

Neste contexto, a homeopatia se enquadra, podendo ser compreendida como uma forma de controle e amenização de disfunções cutâneas com impacto inestético, como é o caso da acne, do melasma, da caspa, entre outras e considerada uma terapia complementar, inclusive com menor número de efeitos adversos relatados.

Em 1997, Linde et al. desenvolveram um estudo visando a verificação de se os resultados obtidos com tratamento homeopático eram devido ao efeito placebo e, após a análise de 89 estudos confiáveis afirmaram que isto não pode ser comprovado, indicando a eficácia desta terapia. Entretanto, os autores apontam para a necessidade de novos estudos, visando maior compreensão e comprovação dos efeitos desta terapia complementar. Neste estudo publicado em 1997, nove trabalhos avaliados empregaram a homeopatia para amenização de desequilíbrios com manifestações dermatológicas e, em 8 deles, haviam indícios de efeitos realmente promovidos pelo tratamento homeopático e não devido ao efeito placebo.

Em 2009 foi publicada uma patente (US20090232904 A1) para o uso de gel homeopático contendo Argentum metallicum para cuidado da pele. Os autores relatam o uso deste produto para diversas finalidades como amenização da irritação da pele, por exemplo, devido à queimadura solar, picadas de inseto, mas também com efeito antimicrobiano, para o tratamento da acne, e de celulite.

Cabe ressaltar ainda que, a população tem desconfiado da segurança de produtos cosméticos e, principalmente, da variada composição dos mesmos, considerando que apesar do uso tópico, podem ser absorvidos pela pele. Existem relatos também da preocupação com a poluição do meio ambiente com a grande quantidade de resíduos promovida pela fabricação, utilização e descarte destes produtos, havendo motivos para a busca de produtos mais simples, menos poluidores e eficazes (CSORBA; BOGLEA, 2011). Estes são alguns dos motivos que tem estimulado a procura, pela população, destas terapias alternativas e complementares (WOODWARD, 2005).

Portanto, este trabalho teve como objetivo realizar uma revisão da literatura buscando embasamento científico para o emprego da homeopatia para amenização de disfunções cutâneas que promovem impacto inestético. Este trabalho visou também apresentar a teoria homeopática e seu fundamento; descrever as principais formas farmacêuticas de uso cosmético que podem ser empregadas para aplicação tópica da homeopatia e, finalmente, correlacionar as principais disfunções cutâneas que promovem impacto 
estético com os insumos ativos que poderiam ser utilizados.

\section{Metodologia}

Este trabalho foi preparado com base em revisão da literatura, com abordagem teórica qualitativa para responder os objetivos previamente listados. Foram avaliados manuscritos nacionais e internacionais publicados entre 1993 a 2019. O tema da pesquisa foi discutido com base em uma coletânea de informações publicadas anteriormente por renomados autores da área. Evidências científicas sobre o emprego da homeopatia em cosméticos foram explicitadas. As palavras-chave utilizadas para a busca foram: homeopatia, homeopáticas, cosméticos, estética, disfunções, dermatologia, pele, tanto no idioma português quanto inglês. As bases de dados utilizadas foram: Google Acadêmico, Lilacs, Scielo, Pubmed e Science Direct.

\section{RESUltados E Discuss Ão}

\section{TEORIA HOMEOPÁTICA E SEU FUNDAMENTO}

A Organização Mundial da Saúde descreve a homeopatia como sendo uma terapia elaborada no final do século XVIII. Enquadra-se nas "Práticas Não-Convencionais em Saúde” (PNCS), assim como a fitoterapia e a acupuntura (OMS, 2009) sendo que, Jonas et al. (2000) sugerem que a homeopatia é a terapia complementar mais usada no mundo.

De acordo com Teixeira (2007), apesar de ser uma prática secular, ainda é desconhecida ou mal compreendida pela maioria da população, inclusive por profissionais da área da saúde.

A homeopatia como prática não-convencional em saúde vem sendo mais amplamente discutida desde 2006, com a criação da Política Nacional de Práticas Integrativas e Complementares (PNPIC) por meio da Portaria no 971 de maio de 2006. Esta Portaria institui, no Sistema Único de Saúde (SUS), a prática de terapias alternativas, como a homeopatia, mas também a fitoterapia, a acupuntura, entre outras (PORTAL DA SAÚDE).

É uma especialidade médica reconhecida desde 1980 (SALLES, 2008), mas que pode também ser praticada por outros profissionais, como farmacêuticos, dentistas e veterinários. A Portaria $n^{\circ} 633$ de março de 2017 indica os médicos, enfermeiros, dentistas e farmacêuticos como profissionais capazes de aplicar a homeopatia no âmbito das práticas integrativas e complementares (PORTARIA 633/2017).

Utiliza como componentes ativos, denominados mais especificamente como insumos ativos, principalmente substâncias de origem vegetal, mineral e animal, preparadas a partir de técnicas de diluição. As diluições são realizadas em insumos inertes (com diferentes graduações etanólicas e lactose), sendo que quanto maior a diluição maior também é a potencialização do insumo ativo (OMS, 2009). A potencialização é maior uma vez que a cada diluição são realizadas sucussões da formulação homeopática, num processo denominado dinamização, capaz de ativar a energia latente nos insumos ativos (RABANES, 1993; DUTRA, 2011; LIMA et al., 2012), sendo este um dos princípios da homeopatia, ou seja, a manutenção da atividade biológica mesmo após as diluições (MERRELL; SHALTS, 2002; WOODWARD, 2005).

A homeopatia visa estimular as forças curativas do organismo sem a necessidade de altas doses de medicamentos, evitando efeitos adversos (TEIXEIRA, 2007), como um dos pilares da homeopatia, ou seja, o uso de doses mínimas ou infinitesimais (DA SILVA, 1997). Pode ocorrer que, em algumas situações, a diluição é tão elevada que não é possível comprovar analiticamente a presença do componente ativo (OMS, 2009). Tem como base a premissa de que, quando há desequilíbrio da força vital, a doença se instala como resultado (MERRELL; SHALTS, 2002).

Para melhor elucidação do assunto, é válido ressaltar que a força vital está em desequilíbrio nos 
indivíduos doentes e que os medicamentos homeopáticos visam reestabelecer este equilíbrio. Weber (2019) relata que "o homem é um ser composto de um corpo, força vital e espírito. A força vital forma um todo com essa unidade. A saúde é o estado em que a força vital mantém todas as partes do corpo, suas funções e sensações, em atividade harmônica. A doença é o desequilíbrio dessa força vital. A cura ocorre quando a força vital reage ao medicamento aplicado."

Oliveira et al. (2004) relatam que "a homeopatia é uma terapia fundamentada no princípio da similitude, explicado pela teoria que a cura decorre da reação secundária do organismo à substância farmacológica, o chamado efeito rebote, num esforço para manter a homeostase”. Isto se resume no princípio de que "semelhante cura semelhante", utilizando como tratamento uma substância que promova efeitos similares (WOODWARD, 2005). TEIXEIRA (2012) afirma que “essa reação vital, homeostática ou paradoxal do organismo está cientificamente embasada no estudo do efeito rebote das drogas modernas”. A utilização do "semelhante" é baseada em outro pilar da homeopatia, a experimentação no homem são, uma vez que a patogenesia dos medicamentos, corresponde aos sinais e sintomas determinados com a experimentação de determinado insumo ativo no indivíduo saudável (DA SILVA, 1997).

Ainda, mais um pilar é descrito na terapia criada por Hahnemann. Trata-se da utilização do medicamento único. Este pilar indica a utilização de um medicamento por vez, entretanto, na prática homeopática, verifica-se que isto não é uma unanimidade. A escolha de alguns homeopatas, muitas vezes, consiste no emprego de mais de um medicamento, visando atingir a totalidade de sinais e sintomas apresentados pelo indivíduo (DA SILVA, 1997). Alguns autores, ainda, consideram este pilar obsoleto pois, na atualidade, a exposição a vários fatores hostis concomitantemente, dificultam a eleição de um medicamento único que trate os sinais e sintomas integralmente (CARESATTO; OLIVEIRA, 2019).

Tem sido considerada uma terapia efetiva, de baixo custo e segura (OLIVEIRA et al., 2004). A segurança do medicamento homeopático é baseada no uso de doses infinitesimais. Entretanto, este é o mesmo motivo que gera intenso debate em relação a esta terapia, pois seu conceito é contrário ao da farmacologia moderna (OMS, 2009).

Neste momento cabe diferenciar a homeopatia da fitoterapia ou do uso de plantas medicinais de maneira geral. A fitoterapia emprega as plantas como fontes de princípios ativos, em doses facilmente quantificáveis, e encontra muito mais semelhanças com a alopatia (WOODWARD, 2005).

A formulação homeopática pode ser preparada a partir de métodos diferenciados. Entre eles podem ser citados: o método hahnemanniano, o método korsakoviano e o de fluxo contínuo, sendo o primeiro, o mais empregado no Brasil (BRASIL, 2011). O método hahnemanniano pode ser utilizado para preparação das escalas decimal, centesimal, cinquenta milesimal recebendo a indicação $\mathrm{DH}$ (10\% de insumo ativo a cada diluição), CH (1\% de insumo ativo a cada diluição) ou LM (proporção de 1/50.000), respectivamente (BRASIL, 2011).

As preparações homeopáticas podem ser preparadas a partir de insumos ativos solúveis e insolúveis, sendo que quando solúveis, o etanol em diferentes diluições é empregado como principal insumo inerte e, quando insolúvel, a lactose é empregada como insumo inerte principal (BRASIL, 2011).

Outra diferença verificada entre as diluições com insumos ativos solúveis e insolúveis é a forma de dinamização. Tanto na escala centesimal quanto decimal hahnemanniana são empregadas 100 sucussões (agitações vigorosas e ritmadas) no caso de preparação de formulações líquidas. Mas, quando os insumos ativos insolúveis são utilizados, na preparação de formulações sólidas, o método de preparo é a trituração, sendo que cada dinamização é baseada na repetição de triturações por 6 minutos e raspagens por 4 minutos, mais especificamente, com 6 ciclos de triturações e 6 ciclos de raspagens, intercalados com a adição de insumo inerte (lactose) (BRASIL, 2011). 


\section{FORMAS FARMACÊUTICAS DE USO COSMÉTICO PARA EMPREGO EM HOMEOPATIA}

Existem diversas possibilidades de formas farmacêuticas que podem ser empregadas para carrear insumos ativos homeopáticos, incluindo formas farmacêuticas líquidas, sólidas e semissólidas. Mas, os medicamentos homeopáticos na forma de gotas (soluções etanólicas), as doses únicas líquidas e os glóbulos (de sacarose) são os mais tradicionais.

Oliveira et al. (2004), em uma pesquisa sobre a utilização de medicamentos homeopáticos em Curitiba, relatam que a forma líquida em gotas é a mais solicitada, seguida da dose única também líquida e dos glóbulos. Apesar de serem formas farmacêuticas efetivas para carrear o insumo ativo homeopático, são destinadas ao uso oral, visando o efeito sistêmico. Considerando a proposta deste trabalho, de empregar a homeopatia para disfunções de resultado inestético, o uso tópico é sugerido.

Oliveira et al. (2004) também destacam a baixa frequência de utilização das formas farmacêuticas tópicas para carrear a homeopatia, relatando ainda que quando são prescritas, normalmente são associadas ao uso oral. Isto pode ser devido à compreensão de homeopatas de que mesmo as manifestações cutâneas são resultado de um desequilíbrio interno (STIBBE, 1999).

A farmacopeia homeopática brasileira ( $3^{a}$ edição) descreve a incorporação de insumos ativos homeopáticos em cremes, géis, e outras formulações destinadas ao uso tópico. Um primeiro exemplo de formulação de uso externo descrito pela farmacopeia homeopática são os linimentos.

Os linimentos são formulações líquidas, que podem ser de base alcoólica, oleosa ou emulsionada (BRASIL, 2011).

Os pós também são descritos, sendo consideradas formulações que contém como insumo inerte alguma substância ou uma composição pulverizada (BRASIL, 2011).

Os cremes são descritos pela farmacopeia homeopática como formulações compostas por fase aquosa, oleosa e um agente emulsivo (BRASIL, 2011). Nwabudike (2011a) relatou casos de tratamento de psoríase com homeopatia, sendo que os cremes foram empregados em alguns pacientes para carrear o insumo ativo homeopático.

Os géis são caracterizados como "dispersões coloidais predominantemente hidrofílicas constituídas por uma fase sólida e uma líquida, de aspecto homogêneo” (BRASIL, 2011). O uso de géis foi descrito na literatura em um experimento realizado por HILL et al., em 1995, que comprovaram a redução de eritema local após picada de mosquitos com a aplicação de gel homeopático. Van Haselen e Fisher (2000) também recorreram aos géis para tratamento homeopático da osteoartrite dos joelhos.

Os géis e os cremes, além das loções, também foram citados em uma patente para composição homeopática para alívio da dor e como anti-inflamatório local. A formulação tem como insumos ativos a Arnica montana, a Belladona, Rhus toxicodendron e Aesculus hippocastanum (US 5795573 A).

Finalmente, as pomadas podem ser citadas, sendo formulações com aspecto homogêneo e de caráter oleoso ou não (BRASIL, 2011).

A farmacopeia indica que a incorporação do insumo ativo, em quaisquer das formas farmacêuticas citadas acima, deve ser feita na concentração de 10\% (BRASIL, 2011).

Dutra (2011) também apresenta estas formas farmacêuticas de uso externo para preparação de formulações homeopáticas, reafirmando a concentração de $10 \%$ do insumo ativo na potência desejada para a impregnação.

\section{Propostas homeopáticas para tratamento de disfunções CutÂneas Com resultados INESTÉTICOS}

São diversas as alterações cutâneas que promovem o incômodo de seu portador e, até mesmo, efeitos em sua qualidade de vida. As alterações podem ocorrer tanto na pele da face, como a acne, o envelhecimento, 
a presença de telangiectasias e discromias (ALSTER, 1999; HANTASH; MAHMOOD, 2007); quanto podem ser visíveis na pele corporal, como as estrias, a celulite e as pseudofoliculites; e nos anexos cutâneos, como as alopecias e a canície.

Apesar da grande variedade de produtos cosméticos disponíveis no mercado atualmente, a população ainda busca por produtos diferenciados, mais efetivos e seguros. Neste contexto, encontra-se a homeopatia, uma terapia alternativa secular e que se propõe, neste trabalho, que seja empregada para finalidades estéticas, no que poderia ser denominado como cosméticos homeopáticos, pois seriam produtos que utilizariam a tradição dos insumos ativos homeopáticos e poderiam ser associados à tecnologia dos produtos cosméticos, obtendo-se produtos estáveis, com sensorial agradável para o uso do consumidor.

Ainda considerando o anseio dos consumidores por produtos mais naturais, mais seguros e com menores impactos ao ambiente, as cosméticas verde e/ou orgânica poderiam ser utilizadas, oferecendo bases cosméticas naturais para incorporação dos insumos ativos homeopáticos (JOHR; SAHASAKMONTRI, 1998; KIM; CHUNG, 2011).

Um insumo ativo homeopático que pode ser citado como exemplo é a Apis mellifica, um inseto da classe das abelhas (BRASIL, 2011). Quando administrada ao indivíduo saudável, este insumo ativo provoca, na pele, erupção vermelha, urticária, erisipela, ou diversos estados inflamatórios podendo se estender às mucosas. Sobre todo o corpo há uma erupção rugosa e espessa. Posteriormente, é notado um quadro inflamatório. $\mathrm{Na}$ face, provoca congestão por todo o rosto, vermelhidão, inchaço e sensação dolorida, o inchaço é mais frequente ao redor dos olhos, estando principalmente nas pálpebras inferiores (LATHOUD, 2002).

Considerando os efeitos promovidos por este insumo ativo quando administrado no homem saudável, principalmente na pele e na região da face, pode-se sugerir o uso deste insumo ativo na Homeopatia, portanto, em doses infinitesimais, para evitar a sensação de vermelhidão, inchaço e irritação na pele, inclusive, na região da face. Desta forma, este insumo ativo pode ser sugerido para uso em cosmético homeopático após a realização de procedimentos estéticos que promovam irritação e eritema da pele da face, como a depilação, limpeza de pele e, até mesmo, peelings. Pode também, ser considerada uma alternativa para a amenização da irritação provocada na pele após a exposição ao sol.

Seguindo este conceito, a seguir estão listados diversos insumos ativos que, de acordo com matériasmédicas ou repertórios homeopáticos, provocam, no indivíduo saudável, efeitos na pele que podem gerar resultados inestéticos. Com base nestas considerações, dos resultados gerados no indivíduo saudável, é que podem ser sugeridos usos destes insumos ativos por meio da lei dos semelhantes.

Os repertórios e as matérias-médicas são literaturas de referências para os profissionais homeopatas, relatando os efeitos das substâncias com base no princípio homeopático da semelhança (RIBEIRO FILHO, 2010). Com base nesta literatura específica, então, foram extraídas informações da patogenesia de importância para a área estética, com ênfase, principalmente, em acne, psoríase, alopecia, caspa e melasma, condições sobre as quais se encontram relatos nas matérias-médicas. Cabe ressaltar que a literatura destaca os sucessos obtidos pela dermatologia com o uso da homeopatia (STIBBE, 1999; WAISSE-PRIVEN, 2009).

A descrição de ensaio patogenético de Dantas (2017) auxilia na compreensão das informações listadas a seguir: "O ensaio patogenético homeopático (EPH) é um estudo experimental para investigar os efeitos de substâncias potencialmente tóxicas ou patogênicas, diluídas e agitadas serialmente conforme recomendado nas farmacopeias homeopáticas, em voluntários não-pacientes que estejam em boas e estáveis condições de saúde. Os EPHs objetivam produzir informações válidas e úteis pertinentes às mudanças objetivas e subjetivas - mentais, gerais ou locais - que um determinado medicamento homeopático pode provocar em seres humanos aparentemente saudáveis e constituem uma evidência do caráter científico e progressista da homeopatia desde a sua fundação". 
O primeiro dos insumos ativos listados é o Amylenum nitrosum. Allen (1995) relata que em contato com este insumo ativo o rosto fica ruborizado a mais leve emoção. Ocorre rápida dilatação dos vasos sanguíneos e aceleração do pulso. Relata-se que o fluxo sanguíneo é intenso na cabeça e face. Estas condições de ruborização facial, com rápida dilatação de vasos, inclusive devido a mais leve emoção é característica de quadros de rosácea, podendo ser o Amylenum nitrosum, portanto, uma opção de tratamento aos indivíduos acometidos por rosácea.

A rosácea é uma disfunção cutânea, que afeta a face, gera grande desconforto, inclusive estético e é de difícil tratamento. Apesar de sua etiologia ainda ser desconhecida, talvez associada com a presença do microorganismo Demodex follicolorum, sabe-se que alguns fatores são agravantes, como emoções fortes, consumo de bebidas alcóolicas e alimentos quentes e apimentados, exposição ao sol (NWABUDIKE, 2012).

Em um estudo realizado por Nwabudike (2012) voluntários da pesquisa, diagnosticados com rosácea, foram tratados com Lachesis, Lycopodium e Causticum, sendo utilizado apenas 1 insumo ativo para cada indivíduo. Os três casos avaliados apresentaram remissão do quadro com o tratamento homeopático realizado.

Segundo Horvilleur (2016), os insumos ativos homeopáticos, descritos acima, tem várias outras indicações para manifestações que acometem ou que apresentam sinais na pele. Lachesis é indicada também para equimoses espontâneas, púrpura, erisipela e icterícia. Já o Lycopodium é indicado para eczema atópico, prurido das regiões que transpiram, verrugas pedunculadas, furúnculos de repetição, úlceras cutâneas crônicas com eczema em torno e de agravamento das dores durante a noite. Causticum, pode ser usado para grandes verrugas pedunculadas, sensíveis ao toque, podendo sangrar, localizadas preferencialmente nos supercílios, pálpebras, nariz, sob ou em torno das unhas.

O Arsenicum album, por sua vez, é reconhecido por promover secura e descamação da pele, que adquire aspecto azulado, enrugado, branco, com vesículas escuras e dor ardente (ALLEN, 1995). A secura excessiva e descamação da pele, com sensação ardente é comum nos casos de psoríase. A psoríase é uma desordem cutânea, de característica inflamatória, que atinge pele, unhas e juntas. São verificados períodos de manifestação intensa da doença seguidos de período de remissão (NWABUDIKE, 2011a).

Boericke (s/d) também relata para o Arsenicum album a elevada sensibilidade e prurido no couro cabeludo, inclusive com placas circulares, aspereza, coberto com escamas, descrição também semelhante à psoríase.

Foi relatado por Albuquerque (2018), como um estudo de caso, que lesões de pele, mais especificamente líquen plano hipertrófico, regrediram completamente após 15 dias de tratamento com Arsenicum album.

Horvilleur (2016) cita que, considerando os efeitos na pele, além da psoríase, o Arsenicum album, é um insumo ativo indicado para erupção, eczema, líquen plano, pitiríase rosada, urticária, equimoses e petéquias.

O Arsenicum iodatum, em relatos de casos, segundo Jurj (2010), foi capaz de promover significativa melhora em um paciente adulto (acometido de forma extrema) e uma criança (acometida de forma leve), que apresentaram eczema, intensa secura de pele, tendendo a descamar na forma de escamas finas e esbranquiçadas (descamação furfurácea).

Horvilleur (2016) também indica o Arsenicum iodatum, para o alívio de erupções sob forma de grandes escamas brancas, abundantes, que contrastam com o fino pó do Arsenicum album, pápulas secas, rugosas, escamosas, eczema liquenificado e líquen plano.

O Antimonium crudum provoca espessamento da pele sendo indicado para o tratamento da acne e de eczemas (VANNIER, s/d). Estas informações estão de acordo com os relatos de Horvilleur (2016), que aponta o Antimonium crudum para hiperqueratoses da pele. Este mesmo autor faz outras indicações de uso deste insumo ativo, como para líquen plano, verrugas córneas da palma das mãos ou da planta dos pés, calosidades da planta dos pés, sensíveis ao choque, unhas espessas, quebradiças, fendidas e que não crescem bem. 
Berberis vulgaris também é descrita por Vannier (s/d) para a amenização de acne, eczema e herpes.

A Bovista lycoperdon, de acordo com Vannier (s/d) gera, entre outros efeitos, odor axilar semelhante à cebola, que também é relatado por Boericke (s/d), além de erupções pruriginosas, crostosas e úmidas na pele. Desta forma, poderia ser utilizada em casos de bromidrose e dermatite seborreica. $\mathrm{O}$ tratamento da dermatite seborreica foi sugerido aqui, uma vez que é uma manifestação cutânea, caracterizada pela hipersecreção sebácea (NWABUDIKE, 2011b).

Nwabudike (2011b) relata o tratamento de indivíduos acometidos por dermatite seborreica com Ignatia, Magnesia carbonica, obtendo resultados satisfatórios.

A Cantharis gera o aparecimento de vesículas por todo o corpo, dolorosas e supurativas (ALLEN, 1995), sendo que uma sugestão seria seu uso para a amenização de quadros de acne nódulo-cística, em que há presença de pus e característica dolorosa. Allen (1995) também a recomenda para aplicação em eritema causado por exposição ao sol. Este autor relata que a Cantharis é capaz de prevenir o surgimento de bolhas e vesículas se aplicada prontamente por via tópica, podendo ser utilizada na formulação de loções pós-sol.

Horvilleur (2016) reforça que Cantharis realmente é indicada para a pele que apresenta lesões cutâneas sob forma de grandes vesículas ou de bolhas, de flictenas, com prurido e sensação de queimação, alergia ao sol.

A Cimicifuga racemosa causa manchas faciais em mulheres jovens, sendo uma possibilidade para amenização de melasmas (BOERICKE, s/d). O tratamento de melasma utilizando Sepia também já foi descrito na literatura (STIBBE, 1999).

O Croton tiglium gera intenso prurido na pele, sendo verificado eczema por todo corpo, com pele muito sensível (ALLEN, 1995). Desta forma, poderia ser recomendado para casos de eczemas.

Dolichos pruriens é indicado para casos em que há acne hipertrófica do nariz, com vermelhidão da pele, acompanhada de varicosidades no nariz e nas bochechas. Vasos sanguíneos temporariamente visíveis e tortuosos (VANNIER, s/d). Estas características são muito semelhantes às da rosácea, incluindo de sua complicação que gera deformidades na região nasal, denominada rinofima.

A Dulcamara torna a pele altamente sensível, inclusive ao frio (agravo em climas frios e amenização no calor), assim como ocorre nos quadros de rosácea. Podem haver erupções, urticária, formação de crostas amareladas com bordas vermelhas que sangram se coçadas (ALLEN, 1995).

O Fluoricum acidum promove efeitos sobre a pele e anexos, inclusive, erupções. De acordo Vannier (s/d) é responsável por unhas quebradiças e deformadas, além de cabelos secos e que caem com facilidade. Desta forma, na Matéria Médica de Vannier, uma de suas indicações é para alopecias.

Também para o Graphites há relatos de que, na cabeça, promove alopecia, cabelos secos e quebradiços e erupções exsudativas (HORVILLEUR, 2016), podendo, portanto, ser outra opção para a queda de cabelos.

Ainda para o Graphites, relata-se que seu uso seja adequado para mulheres propensas à obesidade e que apresentam menstruação atrasada (ALLEN, 1995). Entre outros efeitos, ele promove unhas quebradiças, deformadas e que se esfarelam (ALLEN, 1995). O ganho de peso, associado com atrasos menstruais e unhas quebradiças é relatado em disfunções hormonais, como o hipotireoidismo, a síndrome dos ovários policísticos, etc. Apesar de serem disfunções que promovem efeitos muito além dos estéticos, foram citadas neste trabalho considerando o incômodo gerado nas mulheres acometidas em relação à aparência.

O Kalium arsenicosum, segundo Vannier (s/d) pode ser empregado para psoríase, pois causa uma afecção cutânea crônica, com formação tumoral. Pode causar erupções secas e escamosas, além de prurido intenso. Horvilleur (2016) concorda, e complementa que tem indicações clínicas para líquen plano.

O Kali bromatum é recomendado para pessoas corpulentas, que tendem a ser obesas. Sugere-se que seja mais efetivo em crianças do que em adultos. Causa o surgimento de lesões de acne simples e endurecida, além de rosácea pustular no rosto, peito e ombros (ALLEN, 1995). Considerando os efeitos de Kali 
bromatum no indivíduo saudável, seu uso pode ser indicado para o tratamento de acne, inclusive de comedões, além de rosácea pustular.

O Kali sulphuricum provoca calvície em áreas circunscritas, além de caspa e micose no couro cabeludo. Verifica-se na pele a presença de psoríase, eczema pruriginoso e/ou urticária (BOERICKE, s/d). Há ainda, indicações clínicas para dermite seborreica (HORVILLEUR, 2016).

O Lycopodium clavatum, de acordo com Boericke (s/d), promove formação de manchas marrons, com sardas mais intensas do lado esquerdo da face e do nariz. Desta forma, sugere-se seu uso para melasma, efélides, Thomaz (2009) relata que no vitiligo, a utilização de mica pode induzir a repigmentação. Isto foi observado em um relato de caso, descrito pelo autor previamente citado, de uma única lesão, localizada no queixo, que segundo ele, se justifica por dois sintomas descritos para a patogenesia deste insumo ativo: "descoloração branca da pele" e "descoloração branca da pele em pequenas áreas". Em relação ao vitiligo, Mahesh et al. (2017) relataram que em 14 pacientes tratados com homeopatia individualizada, os melhores resultados foram alcançados nos pacientes que foram tratados nos estágios iniciais de sua doença.

O Natrum muriaticum, sal comum, promove queda dos cabelos quando tocados. O rosto apresenta-se excessivamente oleoso e brilhante. Os lábios são secos, doloridos, rachados, ulcerados. Eczema e urticária no corpo todo, mas principalmente ao redor do couro cabeludo (ALLEN, 1995). Diversos são os tipos de disfunções cutâneas que promovem o eczema generalizado. Desta forma, é possível avaliar o indivíduo e, na presença de outras características, como o excesso de oleosidade da face e as rachaduras dos lábios, o Natrum muriaticum poderia ser recomendado.

Vannier (s/d) indica que o Natrium muriaticum pode ser usado na clínica de alopecia e psoríase também. Horvilleur (2016) relata que tem indicações clínicas para acne, alergia ao sol, alopecia frontal precoce de origem genética, dermite seborreica, eczema, edema de quincke, líquen plano, micose, terçóis, urticária e verrugas.

Relata-se que o Phosphorus gere queda de cabelos em tufos, seguido de calvície em áreas isoladas e caspa excessiva (ALLEN, 1995). Esta queda de cabelos em tufos, com calvície em áreas isoladas é denominada alopecia areata, uma situação muito incômoda ao acometido, que gera inclusive distúrbios psicológicos, em que poderia, portanto, ser empregado loções tópicas de Phosphorus.

O Psorinum, por sua vez, é indicado para indivíduos que apresentam grande sensibilidade ao frio ou às mudanças climáticas. Na pele, verificam-se erupções secas, escamosas que desaparecem no verão e retornam no inverno (ALLEN, 1995). No inverno é muito comum a procura por dermatologistas, esteticistas e a queixa de pacientes sobre o excessivo ressecamento da pele. Nesta pele, uma possibilidade interessante seria a utilização de Psorinum na composição de formulações hidratantes. ALLEN (1995) relata que o Psorinum pode ser usado quando o Sulphur falha em aliviar a condição de ressecamento da pele.

Há também o relato de que o Psorinum provoca ressecamento e ausência de brilho nos cabelos que, inclusive, embaraçam e grudam com facilidade. Também pode causar acne e rosácea, além de ressecamento da pele, com sudorese rara e aparência suja, oleosa (ALLEN, 1995). Muitas vezes, estas características são verificadas em adolescentes que, durante a puberdade, apresentam todas estas características relacionadas à secreção de androgênios.

A Sanicula pode ser indicada para loções anticaspa, pois, de acordo com Allen (1995) promove o surgimento de caspas profusas e escamosas no couro cabeludo, sobrancelhas e barba. Boericke (s/d) também relata, para este insumo ativo, a pele com aspecto de suja e oleosa, além da presença de eczema.

Como pode ser notado, alguns dos insumos ativos homeopáticos descritos são indicados para a amenização do eczema. A pele eczematosa é caracterizada pela presença de ressecamento, eritema, sensibilidade e prurido, em um quadro inflamatório. Desta forma, esta condição pode ser encontrada em casos de dermatite atópica, uma condição crônica que promove desconfortos e efeitos inestéticos 
nos indivíduos acometidos. Uma pesquisa desenvolvida por Itamura e Hosoya (2003) avaliou pacientes com quadro de dermatite atópica diagnosticado e que não haviam obtido melhoras significativas com os tratamentos convencionais.

Cada voluntário da pesquisa recebeu insumos ativos homeopáticos diferenciados, de acordo com a anamnese realizada. Entre os insumos ativos utilizados podem ser citados: Pulsatilla, Arsenicum album, Calcarea carbonicum, Nux vomica, Sulphur, Psorinum, Graphites, Natrum muriaticum, entre outros, em associação ou isolados. Todos os pacientes relataram melhora de $50 \%$ do quadro, sugerindo a efetividade da terapia homeopática. Os autores deste estudo reforçam o fato de que manifestações cutâneas, como a dermatite atópica, geram consequências, inclusive para a qualidade de vida do paciente e para seus aspectos psicossomáticos, por ser a pele um órgão aparente no organismo.

A Sarsaparilla causa erupção por exposição ao ar livre. São erupções secas como sarna que tornam-se crostosas, propensas a surgir na primavera (ALLEN, 1995). A pele se apresenta endurecida e craquelada, com fissuras nas mãos (HORVILLEUR, 2016).

O Selenium metallicum, de acordo com Boericke (s/d) promove seborreia na pele, com presença de comedões, além de alopecia. Conforme Horvilleur (2016), tem como principais indicações clínicas a acne, alopecia e dermite seborreica.

O Sulphur, já descrito acima para ser utilizado antes do Psorinum, torna a pele pruriginosa, com a presença de furúnculos em grupo (ALLEN, 1995). Vannier (s/d) relata que o Sulphur (enxofre) promove ardor na pele com a presença de erupções, de pontos negros sobre o nariz, a testa e o queixo, sendo desta forma, indicado para o tratamento de acne, além de diversas outras disfunções. Jurj (2012) descreve o emprego do sulphur para tratamento de peles acneicas. Horvilleur (2016) também aponta que, na face, promove acne dolorosa, com numerosos cravos e vermelhidões da pele do rosto, sendo o principal insumo ativo para a seborreia gordurosa do rosto.

A Thuja occidentalis promove efeitos na pele e cabelos. Os cabelos tornam-se secos e caem com facilidade. Há presença de caspa. A pele apresenta sardas e manchas (BOERICKE, s/d).

O Tuberculinum promove eczema sobre todo o corpo. O prurido se torna intenso ao se despir, por se banhar. Verificam-se escamas que esfarelam, além de exsudação atrás dos ouvidos, nos cabelos, dobras de pele, pele avermelhada e dolorida. Estas características são comuns em indivíduos acometidos pela psoríase (ALLEN, 1995). Outras indicações clínicas são para alergia, micoses e pitiríase rósea (HORVILLEUR, 2016).

Apesar de não estar relacionada ao uso estético/cosmético, a Calendula não pode deixar de ser citada para o uso dermatológico. É útil em cortes e ferimentos limpos, favorecendo a cicatrização e evitando a supuração (ALLEN, 1995).

Para finalizar, é importante destacar que a homeopatia objetiva cuidar do indivíduo como um todo, sendo que cada pessoa pode receber tratamentos diferenciados de acordo com a anamnese realizada (STIBBE, 1999; ITAMURA; HOSOYA, 2003). Ainda, neste contexto, é preciso considerar a possibilidade de outros órgãos apresentarem sinais de desequilíbrio, sendo que o tratamento da pele e anexos pode não representar o tratamento integral. Neste sentido, estes produtos de uso tópico seriam uma oportunidade de oferecer um cuidado mais direcionado como parte de um tratamento global.

Também não se pode eliminar a possibilidade de associação com outros tratamentos convencionais, visando à melhoria do quadro do indivíduo (STIBBE, 1999).

\section{CoNCLUSÃo}

A homeopatia apresenta diversas vantagens, entre elas podem ser citadas: a menor possibilidade de efeitos adversos e não ser uma terapia invasiva. Apesar das diversas vantagens, seu uso ainda é considerado por muitos apenas como medicamentoso. Entretanto, diversas patogenesias descritas para insumos ativos 
homeopáticos apresentam efeitos promovidos na pele e anexos, podendo, portanto, ser empregada em cosméticos homeopáticos, visando o tratamento ou amenização de disfunções cutâneas que promovem efeitos inestéticos, assim como relatado neste trabalho.

Em suma, uma nova abordagem homeopática foi sugerida neste trabalho, visando estimular estudiosos da área e homeopatas a considerar o uso desta terapia para a amenização de distúrbios estéticos, inclusive por meio de formas farmacêuticas de uso tópico, gerando produtos que poderiam ser denominados como cosméticos homeopáticos, capaz de oferecer um cuidado mais direcionado como parte de um tratamento global.

\section{REFERÊNCIAS}

ALBUQUERQUE, L.V.C. Tratamento homeopático nas dermatoses crônicas do tipo líquen: estudo de caso revisitado após dez anos. Revista de Homeopatia, v.81, n.3/4, p.25-31, 2018.

ALLEN, H.C. Sintomas-chave da matéria médica homeopática: keynotes. São Paulo: Dynamis Editorial, 1995.

ALSTER, T.S. Cutaneous resurfacing with $\mathrm{CO}_{2}$ and erbium: YAG lasers-preoperative, intraoperative, and postoperative considerations. Plast Reconstr Surg, v.103, p. 619-32, 1999.

BOERICKE, W. Materia médica homeopática. Disponível em: http://www.ucipfg.com/Repositorio/FCS/ DIP_MH/DTH-05/UNIDAD3/BOERICKE.pdf. Acesso em julho de 2017.

BRASIL, Farmacopeia Homeopática Brasileira, 3a edição, 2011. Disponível em: http://portal.anvisa. gov.br/documents/33832/259147/3a_edicao.pdf/cb9d5888-6b7c-447b-be3c-af51aaae7ea8. Acesso em fevereiro de 2020.

CARESATTO, C.T.; OLIVEIRA, F.A.A. Farmacotécnica alopática e homeopática: do conhecimento à realização. São Paulo: Editora Senac São Paulo, 2019.

CSORBA, L.M.; BOGLEA, V.A. Sustainable cosmetics: a major instrument in protecting the consumer's interest. Regional and Business Studies, v.3, n.1, p.167-176, 2011.

DANTAS, F. O medicamento homeopático provoca sintomas em voluntários aparentemente sadios? A contribuição brasileira ao debate sobre os ensaios patogenéticos homeopáticos. Revista de Homeopatia, v.80, n.1/2, p.183-206, 2017.

DA SILVA, J.B. Farmacotécnica homeopática simplificada. 2. ed. Piracaia: Robe Editorial, 1997.

DUTRA, V. C. Farmacotécnica homeopática. Rede de Tecnologia e Inovação do Rio de Janeiro REDETEC, 2011.

HANTASH, B. M.; MAHMOOD, M. B. Fractional Photothermolysis: A Novel Aesthetic Laser Surgery Modality. Dermatologic Surgery, v.33, p.525-534, 2007. 
HILL, N.; STAM, C.; TUINDER, S.; VAN HASELEN, R.A. A placebo controlled clinical trial investigating the efficacy of a homeopathic after-bite gel in reducing mosquito bite induced erythema. European Journal of Clinical Pharmacology, v.49, n.1-2, p.103-108, 1995.

HORVILLEUR, A. Vade-Mécum da Prescrição em Homeopatia. ANDREI EDITORA LTDA, 2016.

ITAMURA, R.; HOSOYA, R. Homeopathic treatment of Japanese patients with intractable atopic dermatitis. Homeopathy, v.92, n.2, p.108-114, 2003.

JONAS, W.B.; LINDE, K.; RAMIREZ, G. Homeopathy in rheumatic disease. Rheum. Dis. Clin. North. Am., v.26, p.117-123, 2000.

JOHRI, L.M.; SAHASAKMONTRI, K. Green marketing of cosmetics and toiletries in Thailand. Journal of Consumer Marketing, v.15, n.3, p.265-281, 1998.

JURJ, G. Homeopatia em imagens: Arsenicum iodatum. Revista de Homeopatia, v.73, n.1/2, p.18-30, 2010.

JURJ, G. Homeopatia em imagens: Diagnóstico diferencial da acne, Parte I. Revista de Homeopatia, v.75, n.1/2, p.21-34, 2012.

KIM, H.Y.; CHUNG, J.E. Consumer purchase intention for organic personal care products. Journal of Consumer Marketing, v.28, n.1, p.40-47, 2011.

LATHOUD, J.A. Matéria Médica Homeopática. 5.ed. São Paulo; Robe Editorial, 2002.

LIMA, L.F., ALVES, A.M.C.V.; MAGALHÃES, R.; ROCHA, P.; CELESTINO, J.J.H.; BRUNO, J.B.; RODRIGUES, A.P.R.; FIGUEIREDO, J.R. A homeopatia como alternativa no tratamento de distúrbios reprodutivos. Ciência Animal, v.22, n.2, p.25-43, 2012.

LINDE, K.; CLAUSIUS, N.; RAMIREZ, G.; MELCHART, D.; EITEL, F.; HEDGES, L.V.; JONAS, W.B. Are the clinical effects of homoeopathy placebo effects? A meta-analysis of placebo-controlled trials. Lancet, v.350, p.834-43, 1997.

MAHESH, S.; MALLAPPA, M.; TSINTZAS, D.; VITHOULKAS, G. Case series of vitiligo and homeopathy. Am. J. Case Rep., v.18, p.1276-1283, 2017.

MERRELL, W.C.; SHALTS, E. Homeopathy. Medical Clinics of North America, v.86, n.1, p.47-62, 2002.

NWABUDIKE, L.C. Psoriasis and homeopathy. Proc. Rom. Acad., series B, n.3, p. 237-242, 2011 a.

NWABUDIKE, L.C. Seborrheic dermatitis and homeopathy. Our Dermatol Online, v.2, n.4, p.207-209, 2011b.

NWABUDIKE, L.C. Rosacea and homeopathy. Proc. Rom. Acad., series B, v.14, n.3, p. 207-211, 2012. 
OLIVEIRA, A. B.; ZANIN, S. M. W.; MIGUEL, M. D. A utilização de medicamentos homeopáticos na região metropolitana de Curitiba. Visão Acadêmica, v.5, n.2, p.151 -158, 2004.

OMS. Safety issues in the preparation of homeopathic medicines, 2009. Disponível em: http://apps.who. int/iris/bitstream/10665/44238/1/9789241598842_eng.pdf. Acesso em fevereiro de 2020.

PORTAL DA SAÚDE. Política Nacional de Práticas Integrativas e Complementares em Saúde. Disponível em: http://dab.saude.gov.br/portaldab/pnpic.php. Acesso em junho de 2017.

PORTARIA No 633 de 28 de março de 2017. Disponível em: http://189.28.128.100/dab/docs/portaldab/ documentos/prt_633_28_3_2017.pdf. Acesso em fevereiro de 2020.

RABANES, O. História da homeopatia e do pensamento de Hahnemann. Revista de Homeopatia, v. 58, n.1, p. 4-11, 1993.

SALLES, S.A.C. A presença da homeopatia nas faculdades de medicina brasileiras: resultados de uma investigação exploratória. Revista Brasileira De Educação Médica, v.32, n.3, p.283-000, 2008.

STIBBE, J.R. Homeopathy in dermatology. Clinics in Dermatology, v.17, n.1, p.65-68, 1999.

TEIXEIRA, M.Z. Homeopatia: desinformação e preconceito no ensino médico. Revista Brasileira de Educação Médica, v. 31, n.1, p.15-20, 2007.

TEIXEIRA, M.Z. Homeopatia: prática médica humanística. Rev. Assoc. Med. Bras., v.53, n.6, p.547-549, 2007.

TEIXEIRA, M.Z. Novos medicamentos homeopáticos: uso dos fármacos modernos segundo o princípio da similitude. Revista de Homeopatia, v.75, n.1, p.39-53, 2012.

THOMAZ, L.C.L. Anamnese Homeopática ou Consulta Homeopática? A Propósito do Relato de um Caso de Vitiligo. Revista de Homeopatia, v.72, n.1/2, p.23-29, 2009.

US 5795573 A. Homeopathic pharmaceutical compositions. Disponível em: https://www.google.com/ patents/US5795573. Acesso em fevereiro de 2020.

US 20090232904 A1. Homeopathic Skin Care Compositions and Uses Thereof. Disponível em: https:// www.google.com/patents/US20090232904. Acesso em fevereiro de 2020.

VAN HASELEN, R.A.; FISHER, P.A.G. A randomized controlled trial comparing topical piroxicam gel with a homeopathic gel in osteoarthritis of the knee. Rheumatology (Oxford), v.39, n.7, p.714-719, 2000.

VANNIER, L. Matéria médica homeopática. Disponível em: http://www.ucipfg.com/Repositorio/FCS/ DIP_MH/DTH-03/Unidad-4/lecturas/VannierMateriaMedicaCompleta.pdf. Acesso em julho de 2017.

WAISSE-PRIVEN, S.; JURJ, G.; THOMAZ, L.C.L.; TIERNO, S.A.; LABONIA FILHO, W.; SOS, A.; 
DE SOUZA, M.F.A. Individualized homeopathic treatment of dermatological complaints in a public outpatient clinic. Homeopathy, v.98, p.149-153, 2009.

WEBER, B.T. Vínculos entre homeopatia e espiritismo no Rio Grande do Sul na passagem para o século XX. Hist. Cienc. Saude - Manguinhos, v.26, n.4, p.1299-1315, 2019.

WOODWARD, K.N. The potential impact of the use of homeopathic and herbal remedies on monitoring the safety of prescription products. Human \& Experimental Toxicology, v.24, p.219 -233, 2005. 